\title{
Inibidores da Glicoproteína e Intervenção Coronária Percutânea no Infarto Agudo do Miocárdio: To Be, or Not IIb/IIla, Eis a Questão!
}

\author{
Ver artigo relacionado \\ na página 17
}

\author{
Luiz Alberto Mattos ${ }^{1}$
}

"To be, or not to be: that is the question..."

Hamlet (William Shakespeare, circa 1564-1616)

\begin{abstract}
A reperfusão mecânica no infarto agudo do miocárdio por meio da intervenção coronária percutânea primária (ICP) sobrepujou o tratamento fibrinolítico e, mediante evidências robustas, constituise no tratamento de primeira escolha para pacientes infartados com até 12 horas de diagnóstico e que estão aptos a serem submetidos ao procedimento intervencionista em até 90 minutos do primeiro atendimento. Sua indicação é indiscutível e unânime em todas as principais diretrizes mundiais: é classe I, nível de evidência $\mathrm{A}^{1-3}$.
\end{abstract}

Contudo, as elevadas taxas de sucesso proporcionadas por esse método não estão relacionadas apenas às propriedades mecânicas, intrínsecas a sua concepção, quais sejam a recanalização do vaso pela corda-guia, a lise do trombo oclusivo e o tratamento simultâneo da estenose coronária subjacente por meio da inflação do balão ou, mais modernamente, do implante de stents ${ }^{4}$.

A farmacologia adjunta foi incorporada com maior ênfase a partir dos anos 90 e tornou-se parceira necessária e imprescindível para que o procedimento mecânico seja bem-sucedido, restabelecendo o fluxo coronário anterógrado normal no vaso epicárdico (grau três da classificação do TIMI) e, também, preservando a funcionalidade da microcirculação coronária em mais de $80 \%$ dos pacientes. Sabemos, por meio de estudos comprobatórios angiográficos, que existe relação direta entre a obtenção de altas taxas de reperfusão coronária e a redução da mortalidade tanto imediata como tardia dos pacientes infartados 5 .

A pesquisa original de Sartori et al. ${ }^{6}$ explora, com propriedade, o dilema que se segue, ainda em 2008, acerca da melhor estratégia adjunta para que essa parceria, ICP primária e farmacologia, promova o sinergismo positivo necessário.

$O$ referido artigo promove não apenas uma mas duas reflexões muito pertinentes. A primeira é relacionada à aferição do sucesso do procedimento: a graduação do fluxo coronário epicárdico imediatamente após o procedimento é suficiente? Evidências provenientes de uma série controlada e randomizada demonstram que não.

McLaughlin et al. ${ }^{7}$, em dedicada análise independente, cotejaram o resultado de ICP primária, classificada como bem-sucedida por seus operadores (grau três TIMI) e com o auxílio de central independente, em análise de variáveis angiográficas e eletrocardiográficas de 700 casos. Concluíram que, em somente $62 \%$ dos procedimentos rotulados como "sucesso", se logrou observar a redução do segmento ST elevado. Esses pacientes obtiveram significativas reduções dos desfechos maiores, morte e reinfarto, quando comparados aos demais que obtiveram resolução parcial ou àqueles em que a resolução do ST não se processou $(p=0,001)^{7}$.

Assim, a primeira reflexão dos autores é muito adequada, qual seja, que o sucesso de uma ICP primária aplicada nas primeiras horas do infarto do miocárdio não somente exige que se obtenha o restabelecimento da patência anterógrada epicárdica, mas que essa se acompanhe da resolução rápida e plena do segmento ST, tradução objetiva da preservação da funcionalidade da microcirculação coronária.

Como sempre saliento, o intervencionista dedicado e zeloso na captura do real resultado do procedimento realizado em um paciente infartado deve sem-

\footnotetext{
1 Chefe da Sessão de Pesquisa em Intervenções Percutâneas - Instituto Dante Pazzanese de Cardiologia de São Paulo - São Paulo, SP. Correspondência: Luiz Alberto Mattos. Av. Jandira, 550 - apto. 121 - São Paulo, SP - CEP 04080-003 • E-mail: pivmattos@uol.com.br Recebido em: 31/1/2008 • Aceito em: 6/2/2008
} 
pre visitar a unidade coronária ou de emergência e verificar o eletrocardiograma realizado 90 minutos após a finalização do procedimento. A constatação da regressão plena do segmento ST promoverá a forte certeza de que esse paciente cursará com taxas de mortalidade e reinfarto muito reduzidas, inferiores a $2 \%$, salvo situação de catástrofe hemodinâmica já instalada ou outras morbidades associadas ${ }^{8}$.

A segunda reflexão está relacionada à primeira: para obtermos a plena resolução do segmento ST, rótulo e garantia de reperfusão ótima, é necessário utilizar os inibidores da glicoproteína Ilb/IIla em todos os pacientes? Essa pergunta persegue até hoje uma resposta definitiva, como o Príncipe Hamlet persegue o assassino de seu pai.

Nos primórdios do procedimento, no início dos anos 80, somente estavam disponíveis a aspirina e a heparina não-fracionada. $\mathrm{O}$ advento dos inibidores da glicoproteína IIb/IIla, apresentados como "uma superaspirina endovenosa", foi festejado e recebido com entusiasmo pela comunidade médica, baseado em sua ação farmacológica única, o bloqueio completo e eficaz da via final da agregração plaquetária obtido de modo rápido e pleno após sua administração9

O conhecimento adquirido, até então, sinalizava a importante atuação dos agregados plaquetários, formados in situ em resposta à ruptura da placa aterosclerótica, no risco de promoverem embolização distal e formação de plugs obstrutivos, reduzindo ou paralisando a drenagem arteriovenosa coronária. A disponibilidade dessa nova família de fármacos endovenosos, que pudessem ser utilizados adjuntos à ICP no infarto agudo do miocárdio, tornou-se assim muito sedutora $^{9}$.

Contudo, no contexto da ICP primária, é relevante assinalar que a segurança e a eficácia desses inibidores não foram até hoje respaldadas conclusivamente por qualquer estudo, em termos da redução de eventos clinicamente relevantes, como a mortalidade e o reinfarto. Nesse cenário, não só o abciximab foi o mais estudado, mas, também, o tirofiban e o eptifibatide foram avaliados em investigações de pequeno porte, analisando apenas eventos substitutos dos desfechos clinicamente relevantes.

No estudo CADILLAC, a administração do abciximab foi avaliada em associação à agregação plaquetária dupla (aspirina e ticlopidina) e não mostrou diminuição dos eventos maiores de interesse, como mortalidade ou reinfarto, reduzindo apenas a incidência de trombose subaguda dos stents e a necessidade da realização de outro procedimento de revascularização emergencial para o vaso-alvo ${ }^{10}$.

De acordo com metanálise publicada em 2005, englobando 11 estudos controlados e mais de $27 \mathrm{mil}$ pacientes, que incluiu os estudos RAPPORT, ISAR-2,
CADILLAC, ACE e ADMIRAL, entre outros, o emprego de abciximab mostrou-se eficaz em reduzir a mortalidade de $3,4 \%$ para $2,4 \%(p=0,047)$, diferença significativa embora limítrofe, com impacto menor que o desejado. A redução de risco absoluto da ordem de $1 \%$ mostra que é necessário tratar 100 pacientes com abciximab para que um óbito seja prevenido ${ }^{11}$.

$\mathrm{Na}$ evolução tardia, com seguimento de seis a 12 meses, o gradiente de benefício na redução de mortalidade se alargou um pouco, para 1,8\% (4,4\% vs. $6,2 \% ; p=0,01$ ), sendo necessários 56 pacientes tratados com inibidor da glicoproteína IIb/IIla para prevenção de um óbito ${ }^{11}$. Os mesmos autores, em análise subseqüente, demonstraram uma correlação direta entre a redução da mortalidade e o perfil de risco do paciente, ou seja, maior redução na mortalidade foi encontrada nos pacientes de maior risco ${ }^{12}$.

Sartori et al. ${ }^{6}$ apresentam uma casuística em que não se permite inferir o impacto dessa estratégia farmacológica nos desfechos clínicos maiores, mas concluem, por meio da análise de desfechos substitutos, que a utilização adjunta rotineira de abciximab proporcionou redução mais freqüente do segmento ST, um forte marcador do sucesso do procedimento e preditor independente da redução de mortalidade dos pacientes com IAM submetidos a reperfusão coronária. São freqüentes na literatura séries com casuísticas menores, semelhantes a esta, que não têm o poder estatístico de aferir um desfecho maior como a mortalidade, e que utilizam objetivos secundários ou substitutivos ${ }^{13}$.

Ante os resultados demonstrados, como devemos proceder? A administração rotineira dos inibidores da glicoproteína IIb/IIla, como farmacologia adjunta à ICP primária, não conseguiu obter recomendação mais robusta, e, por isso, sua utilização recebeu, nas mais recentes diretrizes, grau Ila, com nível de evidência B. Em síntese, pode ser utilizada, mas seu uso não é obrigatório ${ }^{3}$.

Se perguntarmos aos operadores que praticam com freqüência esse tipo de procedimento emergencial nos pacientes com infarto agudo do miocárdio, a resposta da maioria é que sua prescrição rotineira traz resultado benéfico ao procedimento, traduzido por maior rapidez no restabelecimento do fluxo coronário tanto epicárdico como distal, facilitando o procedimento mecânico. Opinião certamente carreada de subjetivismo e obtida da prática clínica diária, caso a caso. A Medicina atual, porém, não encontra mais respaldo em impressões isoladas, sem a devida comparação entre as diversas terapêuticas disponíveis e a comprovação inequívoca de benefícios. Assim, sua prescrição tem decrescido, segundo os registros contemporâneos que fotografam a prática clínica da Cardiologia Intervencionista brasileira, em decorrência da falta de evidências robustas e do custo elevado ${ }^{14}$.

A morte ronda o Príncipe Hamlet durante todos os atos da mais popular das peças teatrais de William 
Shakespeare. Da mesma forma, essa é a nossa preocupação principal no tratamento dos pacientes com IAM, ao instituirmos o melhor tratamento possível e, assim, tentar reduzir ao máximo seu risco de óbito ${ }^{15}$.

Parafraseando o famoso dramaturgo e poeta inglês, é necessário que novas investigações verifiquem esses resultados, como o Príncipe Hamlet filosofa com seu amigo Horácio: "Há mais coisas no céu e na terra, Horácio, do que sonha tua filosofia". É o que eu diria a Sartori et al. ${ }^{6}$.

Intervenções em infartados devem obter consistente redução da mortalidade com custo condizente, para serem incorporadas de modo universal à prática clínica, ou então, como diria novamente o Príncipe Hamlet a Horácio, "o resto é silêncio".

\section{REFERÊNCIAS BIBLIOGRÁFICAS}

1. Keeley EC, Boura JA, Grines CL. Primary angioplasty versus intravenous thrombolytic therapy for acute myocardial infarction: a quantitative review of 23 randomised trials. Lancet. 2003;361(9351):13-20.

2. Piegas LS, Timerman A, Nicolau JC, Mattos LA, Rossi Neto JM, Feitosa GS. III Diretriz sobre tratamento do infarto agudo do miocárdio. Arq Bras Cardiol. 2004;83(supl IV):1-86.

3. King SB $3^{\text {rd }}$, Smith SC Jr, Hirshfeld JW Jr, Jacobs AK, Morrison DA, Williams DO, et al. 2007 focused update of the ACC AHA/SCAI 2005 guideline update for percutaneous coronary intervention: a report of the American College of Cardiology/American Heart Association Task Force on Practice Guidelines. J Am Coll Cardiol. 2008;51(2):172-209.

4. Costantini CO, Stone GW, Mehran R, Aymong E, Grines $\mathrm{CL}, \mathrm{Cox} \mathrm{DA}$, et al. Frequency, correlates, and clinical implications of myocardial perfusion after primary angioplasty and stenting, with and without glycoprotein Ilb/IIla inhibition, in acute myocardial infarction. J Am Coll Cardiol. 2004;44(2):305-12.

5. The GUSTO Angiographic Investigators. The effects of tissue plasminogen activator, streptokinase, or both on coronaryartery patency, ventricular function, and survival after acute myocardial infarction. N Engl J Med. 1993;329(22):1615-22.

6. Sartori C, Manica A, Sarmento-Leite R, Heineck G, Almeida G, Quadros A, et al. Papel dos inibidores da glicoproteína
Ilb/IIla na resolução do segmento ST em pacientes submetidos a angioplastia primária. Rev Bras Cardiol Invas. 2008;16(1):17-23.

7. McLaughlin MG, Stone GW, Aymong E, Gardner G, Mehran R, Lansky AJ, et al. Prognostic utility of comparative methods for assessment of ST-segment resolution after primary angioplasty for acute myocardial infarction: the Controlled Abciximab and Device Investigation to Lower Late Angioplasty Complications (CADILLAC) trial. J Am Coll Cardiol. 2004;44(4):1215-23.

8. Boden WE, Eagle K, Granger CB. Reperfusion strategies in acute ST-segment elevation myocardial infarction: a comprehensive review of contemporary management options. J Am Coll Cardiol. 2007;50(10):917-29.

9. Brener SJ, Barr LA, Burchenal JE, Katz S, George BS, Jones $\mathrm{AA}$, et al. Randomized, placebo-controlled trial of platelet glycoprotein IIb/IIla blockade with primary angioplasty for acute myocardial infarction. ReoPro and Primary PTCA Organization and Randomized Trial (RAPPORT) Investigators. Circulation. 1998;98(8):734-41.

10. Stone GW, Grines CL, Cox DA, Garcia E, Tcheng JE, Griffin $\mathrm{J}$, et al. Comparison of angioplasty with stenting, with or without abciximab, in acute myocardial infarction. $N$ Engl J Med. 2002;346(13):957-66.

11. De Luca G, Suryapranata H, Stone GW, Antoniucci D, Tcheng JE, Neumann FJ, et al. Abciximab as adjunctive therapy to reperfusion in acute ST-segment elevation myocardial infarction: a meta-analysis of randomized trials. JAMA. 2005;293(14): 1759-65.

12. De Luca G, Suryapranata H, Stone GW, Antoniucci D, Tcheng JE, Neumann FJ, et al. Relationship between patient's risk profile and benefits in mortality from adjunctive abciximab to mechanical revascularization for ST-segment elevation myocardial infarction: a meta-regression analysis of randomized trials. J Am Coll Cardiol. 2006;47(3):685-6.

13. Maioli M, Bellandi F, Leoncini M, Toso A, Dabizzi RP. Randomized early versus late abciximab in acute myocardial infarction treated with primary coronary intervention (RELAxAMI Trial). J Am Coll Cardiol. 2007;49(14):1517-24.

14. Mattos LA, Sousa AG, Pinto IM, Silva ER, Carneiro JK, Sousa JE, et al. A comparison of rescue and primary percutaneous coronary interventions for acute myocardial infarction: a multicenter registry report of 9,371 patients. Arq Bras Cardiol. 2004;82(5):440-4.

15. Nallamothu BK, Bradley EH, Krumholz HM. Time to treatment in primary percutaneous coronary intervention. $\mathrm{N}$ Engl J Med. 2007;357(16):1631-8. 